\title{
Validity of fruit and vegetable intake assessed by a food frequency questionnaire (FFQ) in older adults: the Northern Ireland Cohort for the Longitudinal Study of Aging (NICOLA)
}

\author{
C.E. Neville ${ }^{1}$, M.C. McKinley ${ }^{1}$, F. Kee ${ }^{1}$, I.S. Young ${ }^{1}$, C.R. Cardwell ${ }^{1}$ and J.V. Woodside ${ }^{1}$ \\ ${ }^{1}$ UK CRC Centre of Excellence for Public Health, School of Medicine, Dentistry and Biomedical Sciences, Queen's \\ University Belfast, Belfast, BT12 6BJ
}

Accurately assessing dietary intake in older populations is vital to determine the potential role of diet in healthy ageing. However, accurate estimation of dietary intake is difficult, with commonly employed methods each being associated with error ${ }^{(1)}$. Assessing dietary intake in older populations can also be challenging as individuals may not be involved in their own food preparation, may not be physically able to record intakes, or may have cognitive impairments impacting on their ability to accurately recall intake. It is therefore essential that commonly-used dietary assessment methods are validated in older populations. Based on the uncertainty over the utility of a food frequency questionnaire (FFQ) to determine dietary intake in older people, the objective of this study was to assess the relative validity of assessing fruit and vegetable (FV) intake from a FFQ compared with a food diary (FD).

A sub-sample of 50 participants (aged >50 years) from the Northern Ireland Cohort for the Longitudinal Study of Aging (NICOLA) completed a FFQ and 4-day FD at two time-points (Month 0 and Month 6). Estimates of FV intake were compared between methods using Spearman's correlation coefficients, examining the percentage of participants classified into the same or adjacent quartile of FV intake, weighted kappa and Bland-Altman plots.

At both time-points, median fruit, vegetable and total FV intake were significantly higher in the FFQ than the FD. Significant positive correlations (all p < 0.05) were observed between the FFQ and FD estimates of FV intake at both time-points (Month 0, $\mathrm{r}=0.44,0.52$ and 0.46 for fruit, vegetables, total $\mathrm{FV}$, respectively; Month $6 \mathrm{r}=0.49,0.44$ and 0.44 , respectively). After individuals were put in fourths (based upon quartiles of total FV portions by FD or FFQ), $79 \%$ and $85 \%$ of participants were classified into the same or adjacent quartile at Month 0 and Month 6, respectively, while weighted kappa showed fair-moderate agreement between methods for FV intake (weighted kappa $=0.33$ and 0.44 at Month 0 and Month 6 , respectively). Bland-Altman plots revealed a widening in limits of agreements, between the FFQ and FD, with higher FV intakes. Significant positive correlations were observed between total FV intakes reported at Month 0 and those reported at Month $6(\mathrm{r}=0.53, \mathrm{p}<0.001)$, with correlation coefficients being stronger for fruit intake $(\mathrm{r}=0.65, \mathrm{p}<0.001)$ compared to vegetable intake $(\mathrm{r}=0.36, \mathrm{p}=0.01)$.

\begin{tabular}{|c|c|c|c|c|c|c|}
\hline & & \multicolumn{2}{|c|}{ FFQ } & \multicolumn{2}{|c|}{ Food Diary } & \multirow[t]{2}{*}{$\mathrm{p}$ value $\mathrm{a}^{\mathrm{a}}$} \\
\hline & & Median & IQR & Median & IQR & \\
\hline \multirow[t]{3}{*}{ Month 0} & Fruit intake (portions/d) & 3.8 & $1 \cdot 6,5 \cdot 2$ & $1 \cdot 3$ & $0 \cdot 7,2 \cdot 1$ & $<0 \cdot 001$ \\
\hline & Vegetable intake (portions/d) & $4 \cdot 5$ & $2 \cdot 7,6 \cdot 5$ & 1.6 & $1 \cdot 0,2 \cdot 0$ & $<0 \cdot 001$ \\
\hline & Fruit and vegetable intake (portions/d) & $7 \cdot 9$ & $5 \cdot 3,11 \cdot 1$ & $3 \cdot 1$ & $2 \cdot 2,4 \cdot 3$ & $<0.001$ \\
\hline \multirow[t]{3}{*}{ Month 6} & Fruit intake (portions/d) & $3 \cdot 0$ & $2 \cdot 1,4 \cdot 2$ & $1 \cdot 0$ & $0 \cdot 03,1 \cdot 9$ & $<0.001$ \\
\hline & Vegetable intake (portions/d) & $3 \cdot 7$ & $2 \cdot 6,4 \cdot 9$ & $1 \cdot 8$ & $1 \cdot 2,2 \cdot 5$ & $<0 \cdot 001$ \\
\hline & Fruit and vegetable intake (portions/d) & $6 \cdot 7$ & $4 \cdot 4,9 \cdot 8$ & $2 \cdot 8$ & $2 \cdot 0,4 \cdot 0$ & $<0 \cdot 001$ \\
\hline
\end{tabular}

Values are median and inter-quartile range (IQR). ${ }^{a} \mathrm{p}$ value for difference between FFQ and FD using Wilcoxen signed rank test

While over-reporting is evident with the FFQ compared to the FD, the results show good comparability between the methods in being able to rank older adults according to their FV intake. Analysis of FV biomarkers within this sample will provide a more objective assessment of FV intake by each method.

1. Kipnis V, Midthune D, Freedman L, et al. (2001) Public Health Nutr 5, 915-923. 